\title{
A case report on expanding horizon of endobronchial ultrasound through esophagus
}

\author{
Mario Tamburrini ${ }^{1}$, Parikshit Thakare, ${ }^{2}$ Francesca Zampieri ${ }^{1}$, Angelo Scarda ${ }^{1}$, Alessandra Di Paolo ${ }^{1}$, \\ Giancarlo De Leo, ${ }_{1}^{1}$ Enrico Gianfagna, ${ }^{1}$ Lucia Vietri, ${ }^{1}$ Umberto Zuccon ${ }^{1}$ \\ ${ }^{1}$ Department of Internal and Specialist Medicine, Pneumology Unit, Friuli Venezia Giulia Health Authority Hospital of \\ Pordenone, Italy ${ }^{2}$ Department of Pulmonary Medicine, TNMC \& BYL Nair Ch Hospital, Mumbai, India
}

\begin{abstract}
Endobronchial ultrasound has revolutionized the field of bronchoscopy and has become one of the most important tools for the diagnosis of intrathoracic lymphadenopathy and para-bronchial structures. The reach of this technique has not been limited to these structures and pleural lesions have been at times accessible. To our knowledge, pleural fluid collections have not been accessed with endobronchial ultrasound (EBUS) through oesophageal approach and rationale behind using this approach. We report a case of 70 years old man who has been referred from
\end{abstract}

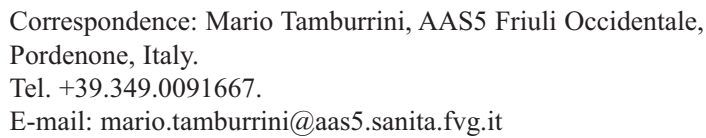

Key words: lymphadenopathy; endobronchial ultrasound through esophagus (EUS-B); thoracocentesis.

Contributions: MT, UZ were involved in patient management; FC, AS, $\mathrm{AD}, \mathrm{GD}, \mathrm{EG}, \mathrm{LV}$ did the literature search and wrote the manuscript. All the authors have read and approved the final version of the manuscript and agreed to be accountable for all aspects of the work.

Funding: The authors do not have specific grant for this research from any funding agency in the public, commercial or not-for-profit sectors.

Conflict of interest: The authors declare no conflict of interest.

Informed consent: Written informed valid consent was obtained from the patient.

Consent for publication: The patient gave his written consent to use his personal data for the publication of this case report and any accompanying images.

Received for publication: 17 March 2020.

Accepted for publication: 22 May 2020.

${ }^{\circ}$ Copyright: the Author(s), 2020

Licensee PAGEPress, Italy

Monaldi Archives for Chest Disease 2020; 90:1274

doi: 10.4081/monaldi.2020.1274

This article is distributed under the terms of the Creative Commons Attribution Noncommercial License (by-nc 4.0) which permits any noncommercial use, distribution, and reproduction in any medium, provided the original author(s) and source are credited. physician for the EBUS in view of hilar mass with mediastinal lymphadenopathy with pleural effusion. The endobronchial ultrasound through esophagus (EUS-B) was done for thoracocentesis and lymph node cytology evaluation and ultimately endobronchial biopsy of hilar mass was done as rapid on-site (ROSE) analysis of lymph node was suggestive of necrotic tissue. The cytology report of lymph node and pleural effusion was positive for malignant cells. The final diagnosis was metastatic poorly differentiating adeno-squamous carcinoma.

\section{Introduction}

Endobronchial ultrasound (EBUS) is very useful, accurate and minimally invasive tool for hilar and mediastinal lymph nodes structure assessment using conscious sedation. It has been increasingly used to biopsy other hilar and midline accessible structures $[1,2]$. When an EBUS scope is passed through the esophagus to assess the mediastinal structures, it is termed an endobronchial ultrasound thorough esophagus (EUS-B). It is being frequently used to sample the mediastinal structures through the esophagus [3]. It provides nearly complete access to all relevant lymph nodes for staging lung cancer; permits the diagnosis of para-esophageal mediastinal and lung lesions which cannot be accessed through the tracheo-bronchial tree. A revised staging system for non-small cell lung cancer has recently been proposed [4], where the presence of malignant pleural effusion (PE) has been upgraded from T4 to M1 (invariably Stage IV). Thus obtaining a sample of pleural effusion whenever present for cytological examination to verify or exclude the presence of malignant cells have become two very important steps in the staging of these neoplasms [5]. However there is less literature available for the EUS-B guided thoracocentesis. We report a case where in EUS-B was done for the lymph node biopsy and thoracocentesis eventually turned out to be malignancy.

\section{Case Report}

A 70-year-old hypertensive man presented in emergency department with history of dizziness, headache and nausea with history of dry cough for 20 days, not associated with fever and managed with antibiotics for short time some days ago. There were no complaints of chest pain and breathlessness. The general and respiratory system examination was normal with bilateral equal breath sounds. The recorded vital parameters were pulse- $84 / \mathrm{min}$, oxygen saturation$98 \%$, respiratory rate- $18 /$ minute and blood pressure was $120 / 78 \mathrm{~mm}$ 
Hg. The central nervous examination and ophthalmic examination was normal. The blood reports showed hemoglobin report of 12.6 $\mathrm{gm} / \mathrm{dL}$, total leucocyte count was $20,180 / \mathrm{mm}^{3}$, platelet counts were $72,000 / \mathrm{mm}^{3}$ and $\mathrm{C}$ reactive protein (CRP) was $6.9 \mathrm{mg} / \mathrm{dL}$. The chest$\mathrm{X}$ ray was suggestive of right sided small pleural effusion.

The patient was diagnosed as a case of vertigo under evaluation and mild pneumonia and managed symptomatically in emergency department and planned for otorhino-aryngologist visit on next day. The patient again visited emergency department on next day with symptoms of nausea, vomiting and vertigo. The patient hospitalized under general medicine department and subsequently evaluated with computed tomography of the brain suggestive some hypodense area in the right cortico-subcortical parietal area due to ischemia and contrast enhanced computed tomography (CECT) of thorax was suggestive of right hilar mass lesion 29x24 mm with narrowing of segmental bronchus with bi-lateral hilar and subcarinal lymph node being largest of size $43 \times 27 \mathrm{~mm}$ along with subcentrimetric bi-lateral supraclavicular lymph nodes with left lung multiple nodular opacities predominantly on lower lobe areas with right sided pleural effusion with thickness of $6 \mathrm{~cm}$ and atelectasis of the medial, posterior and lateral basal segments of the right lower lobe with CECT of abdomen suggestive of left adrenal gland nodularity with L3 vertebral bone lysis possibly secondary metastasis.

\section{Diagnostic evaluation}

In view of the above presentation, the physician directly referred the patient for EBUS for the diagnostic evaluation. Doing initial thoracocentesis and then wait for its report and subsequently planning for EBUS study would have been more time consuming, hence EUS-B approach was preferred. The EUS-B guided thoracocentesis was done and around $50 \mathrm{~mL}$ of fluid was removed followed by lymph node aspiration to know the exact nodal staging and finally endobronchial biopsy was taken as lymph node aspiration on ROSE was suggestive of presence of necrotic cells and absence of malignant cells using above described standard method. So, with the use of this technique T,N,M components were evaluated for malignancy in a single setting with more safe and less invasive approach which saved patient's multiple setting visits to higher centre for further evaluation and management.

The pleural fluid characteristics were yellow in colour and turbid in appearance with $\mathrm{pH}$ of 7.8 , protein of $4.2 \mathrm{gm} / \mathrm{dL}$, glucose of $89 \mathrm{gm} / \mathrm{dL}$ and LDH of $593 \mathrm{U} / \mathrm{L}$. The cytology report profile eventually turned out to be positive for malignant cells in fine needle aspiration cytology (FNAC) under ultrasound guidance for subcarinal lymph node and EUS-B guided thoracocentesis. The histopathology profile of hilar mass was also positive for malignant cells showing final diagnosis of the patient was metastatic poorly differentiated adeno-squamous carcinoma with immunophenotypic and histochemical profile suggestive of p40 focal positive, thyroid transcription factor (TTF) was negative, napsin A was negative, cytokeratin (CK7) was positive and Periodic Acid-Schiff with diastase (PASD) was focal positive.

\section{Methods}

A valid written informed consent was taken from the patient. EBUS was performed using a convex probe EBUS Olympus-BFUC180F EBUS scope and the EU-ME2 ultrasound (Olympus).
The procedure was performed by two pulmonologists, a nursing staff and was done under conscious sedation by an anesthetist with a mouth guard using intravenous midazolam and fentanyl as advised by the BTS guidelines for bronchoscopy. Local anaesthesia was achieved by spraying $10 \%$ lignocaine in the posterior pharynx prior to the procedure. The bronchoscope was passed by one bronchoscopist via the mouth guard. The routine bronchoscopy evaluation was done to visualize vocal cords, trachea and carina following which an EBUS study was attempted. It was decided to use EBUS bronchoscope using oesophageal approach (EUS-B) as it was less invasive and more safe approach for the lymph node biopsy and thoracocentesis also. The thoracocentesis was done (Figures 1 and 2) initially (M-Metastasis evaluation) and around $15 \mathrm{~mL}$ of fluid was aspirated for malignant cytology analysis followed by subcarinal lymph node aspiration (NLymph node evaluation) (Figure 3) using standard 22 G Cook's biopsy needle. The lesions were identified using digitally captured ultrasound images. The 22-gauge Olympus needle was used to puncture the lymph nodes and after each pass, slides were prepared and stained them with toluidine blue to identify lymphocytes, granulomas and atypical cells. Up to three passes were made for the lymph node and rapid on-site evaluation (ROSE) was which was suggestive of necrotic cells and absence of any bronchial cells, malignant cells or lymph node tissue. The slides were then air-dried and sent for cytopathological analysis and acid-fast bacilli stain. In the end endobronchial biopsy was taken of the hilar mass (T-Tumour evaluation) using endobronchial biopsy needle for the histopathological evaluation.

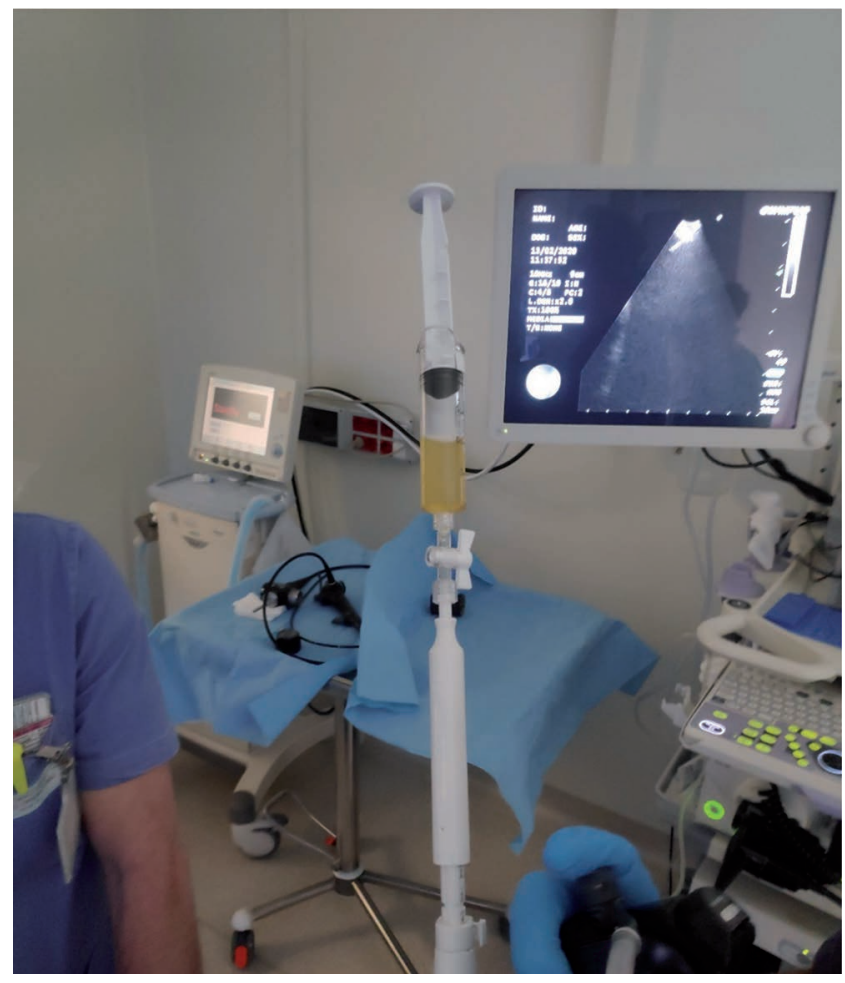

Figure 1. EUS-B guided thoracocentesis. 


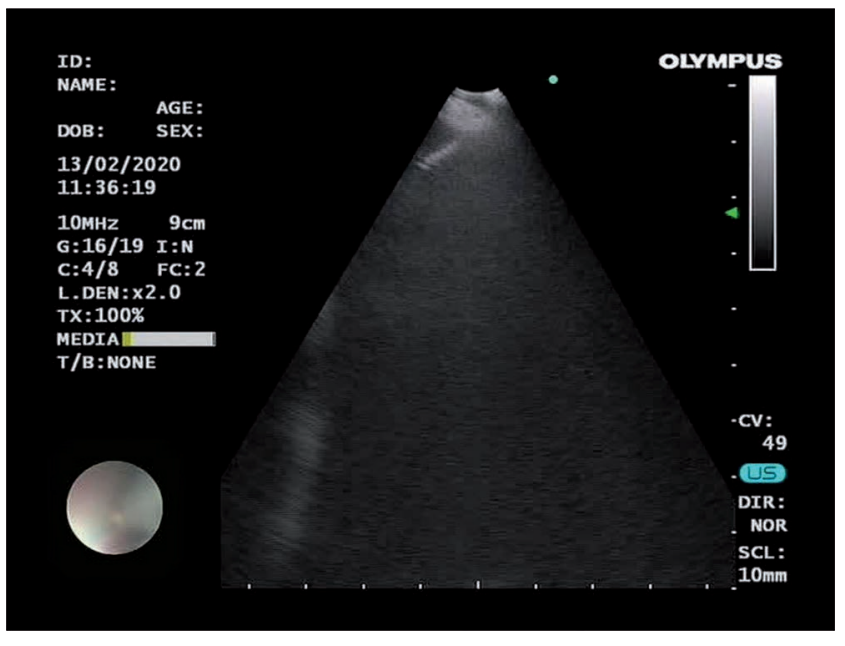

Figure 2. EUS-B guided thoracocentesis (ultra-sonographic view).

\section{Discussion}

The evaluation of the cytology or histopathology sample is very important in cases of suspected lung malignancies. In such cases samples can be obtained using either lung tissue, lymph node or pleural fluid to obtain the diagnosis and achieving TNM staging. For the staging of lung malignancies it is important to obtain good quality sample with the safer and less invasive approach. With the recent advancement in interventional pulmonology, the EBUS or EUS-B has been used to obtain the sample whenever feasible. The patient presenting with suspected malignant pleural effusion used to be evaluated with traditional percutaneous thoracocentesis.

In the literature there are limited data on the utility of EUSguided thoracentesis for the pleural fluid evaluation [6,7]. Chang et al. [6] performed this procedure in two patients with gastric carcinoma to diagnose malignant ascites and malignant pleural effusion not detected by standard radiological examinations. De Witt et al. [7], in a retrospective single-centre study, identified nine consecutive cancer patients who underwent EUS-guided thoracentesis: in two cases the pleural sampling provided the diagnosis of a malignant effusion, while in the remaining seven cases, pleural fluid cytology was negative.

Considering the ease, efficacy and directness of EUS in obtaining suspected lung tissue for malignancy (T-status assessment) and lymph nodes involved (N-status assessment) $[5,8]$ it is believed that EUS may play a significant role in the decision-making process of non small cell lung cancer (NSCLC) management. In selected cases of posterior effusion or patients with comorbidities who could not withstand a thoracentesis procedure or when thoracentesis may not be feasible; EUS-FNA (fine needle aspiration) of pleural effusion (M-status assessment) may represent a very useful tool in the NSCLC clinical staging. The EUS-guided diagnostic thoracocentesis is a safe and effective alternative method for evaluating patients with minimal pleural effusion [9]. The EUS combined with EUSguided FNA can be used for the diagnosis of malignant pleural or ascitic fluid in patients with gastrointestinal malignancies and might further extend the staging capabilities of EUS [10].

The EUS-B has been used in various non malignant diseases to achieve diagnosis like sarcoidosis, tubercular lymphadenitis. The EUS has been used to obtain samples of pericardial effusion,

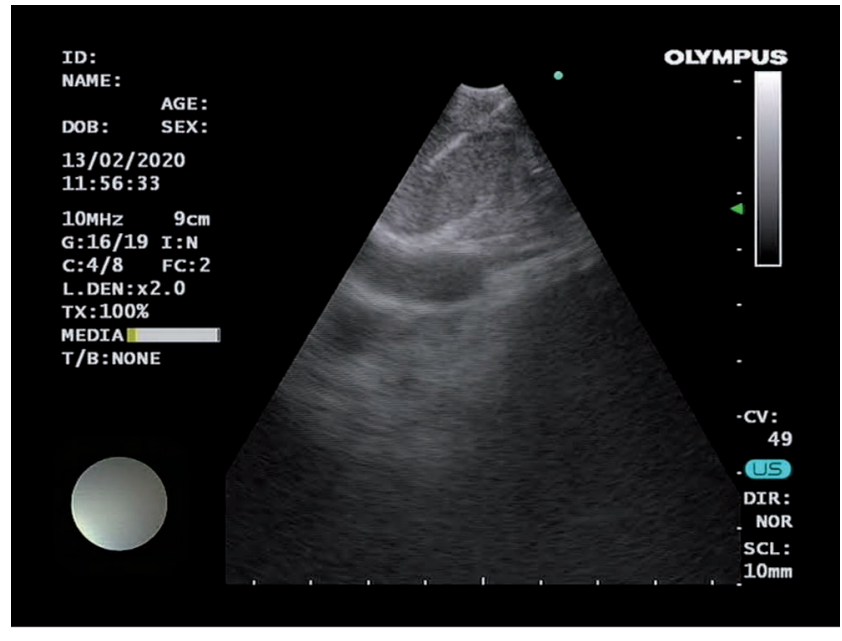

Figure 3. 22-G-Cook's biopsy needle in the subcarinal lymph node.

parathyroid adenoma and thyroid lesion. However there is only a single case has been reported for thoracocentesis using EUS-B [11].

The use of the esophagus is often a better option for some patients with limited respiratory reserve and sometimes the only available option such as the case of a patient intubated with a small endotracheal tube [12].

\section{Conclusions}

Thus EUS-B can be used as a novel, safe and less invasive technique in expert hands wherever such facility is available which can provide good quality samples in a single setting visit for the TNM staging in cases of suspected lung malignancy. Therefore EUS-B can be used for thoracocentesis in expanding its horizon apart from taking biopsy samples of mediastinal structures and lymph node tissue and other to achieve the diagnosis. However, it should not be used routinely as it requires expertise and highly equipped interventional pulmonology setup.

\section{References}

1. Leff AR. Erratum: Endobronchial ultrasound for the diagnosis and staging of lung cancer. Proceed Am Thorac Soc 2009; 1;6:394.

2. Du Rand IA, Barber PV, Goldring J, et al. British Thoracic Society Interventional Bronchoscopy Guideline G. British Thoracic Society guideline for advanced diagnostic and therapeutic flexible bronchoscopy in adults. Thorax 2011;1;66:iiil-21.

3. Dietrich CF, Annema JT, Clementsen P, et al. Ultrasound techniques in the evaluation of the mediastinum, part I: endoscopic ultrasound (EUS), endobronchial ultrasound (EBUS) and transcutaneous mediastinal ultrasound (TMUS), introduction into ultrasound techniques. J Thorac Di. 2015;7:E311.

4. Rami-Porta R, Ball D, Crowley J, et al. The IASLC Lung Cancer Staging Project: proposals for the revision of the T descriptors in the forthcoming (seventh) edition of the TNM classification for lung cancer. J Thorac Oncol 2007;2:593-602. 
5. Varadarajulu S, Schmulewitz N, Wildi SM, et al. Accuracy of EUS in staging of T4 lung cancer. Gastrointest Endosc 2004;59:345-8.

6. Chang KJ, Albers CG, Nguyen P. Endoscopic ultrasound-guided fine needle aspiration of pleural and ascitic fluid. Am J Gastroenterol 1995;90:148-50.

7. DeWitt J, Kongkam P, Attasaranya S, et al. Endoscopic ultrasound-guided transesophageal thoracentesis. Endoscopy 2008;40:E118-9.

8. Annema JT, van Meerbeeck JP, Rintoul RC, et al. Mediastinoscopy vs endosonography for mediastinal nodal staging of lung cancer: a randomized trial. JAMA 2010;304:2245-52.
9. Rana SS, Sharma R, Gupta R. Endoscopic ultrasound-guided transesophageal thoracentesis for minimal pleural effusion. Indian J Gastroenterol 2018;37:231-4.

10. Cocciardi S, Borah A, Terrigno R, et al. A case report of an expensive yet necessary thoracentesis: Expanding the boundaries of endoscopic ultrasound transbronchial needle aspiration. Medicine (Baltimore) 2019;98:e17555.

11. Abouzgheib W, Shweihat Y, Bartter T. Oesophageal applications of the convex curvilinear ultrasound bronchoscope; an illustrative case series. Respirol 2011;16:965-8.

12. Meena N, Hulett C, Jeffus S, Bartter T. Left adrenal biopsy using the convex curvilinear ultrasound scope. Respiration 2015;89:57-61. 